\title{
On the Impact of Aging on Power Analysis Attacks Targeting Power-Equalized Cryptographic Circuits
}

Md Toufiq Hasan Anik ${ }^{1}$, Bijan Fadaeinia ${ }^{2}$, Amir Moradi ${ }^{2}$ and Naghmeh Karimi ${ }^{1}$ ${ }_{1}^{1}$ University of Maryland Baltimore County, USA

${ }^{2}$ Ruhr University Bochum, Horst Görtz Institute for IT Security, Germany

20 January 2021

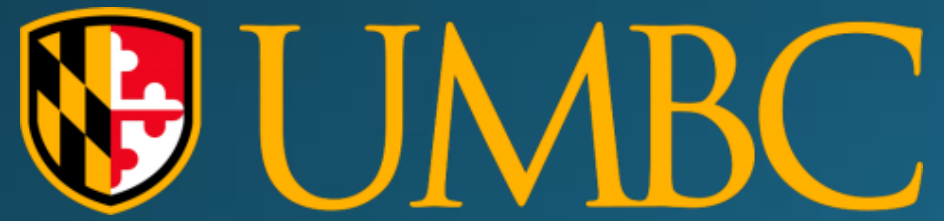

RUB 


\section{Outline}

$\square$ Introduction

- Power Analysis Attacks

- Dual Rail Logics (SABL, ...)

$\square$ Device Aging

$\square$ Targeted Circuit

$\square$ Experimental Results

- Aging-induced Delay Change

- Power Analysis Attacks

$\square$ Conclusion and Future Directions 


\section{Cryptographic Circuits}

口 Preserving data confidentiality via cryptographic circuits

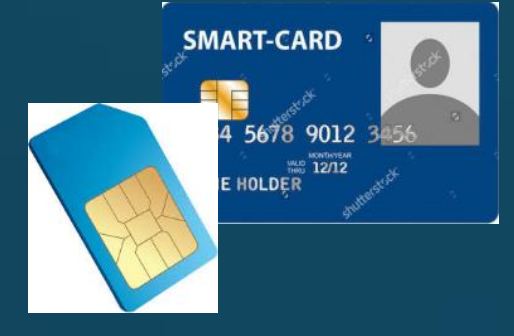

C. 5 The physical implementation can be compromised

- Observing dynamic circuit emanations

- Side Channel Attacks: Analyzing the physical leakage

- Running time

- Electromagnetic radiation

- Power consumption 


\section{Power Analysis Attacks}

Revealing a cryptographic device's key by analyzing its power consumption

$\square$ The key is retrieved based on the dependency of:

- Power leakage and the data being processed

- Power leakage and the operation being performed

口 Countermeasure:

- Hiding: Decreasing Signal-to-Noise ratio (SNR)

- Masking: Randomizing the intermediate values

Hiding Schemes mainly equalize the power consumption regardless of the data being processed

- Dual-Rail Pre-Charge (DRP):

- Complementary Counterpart

- Fed with the primary inputs \& their complements

- Generates the outputs \& their complement

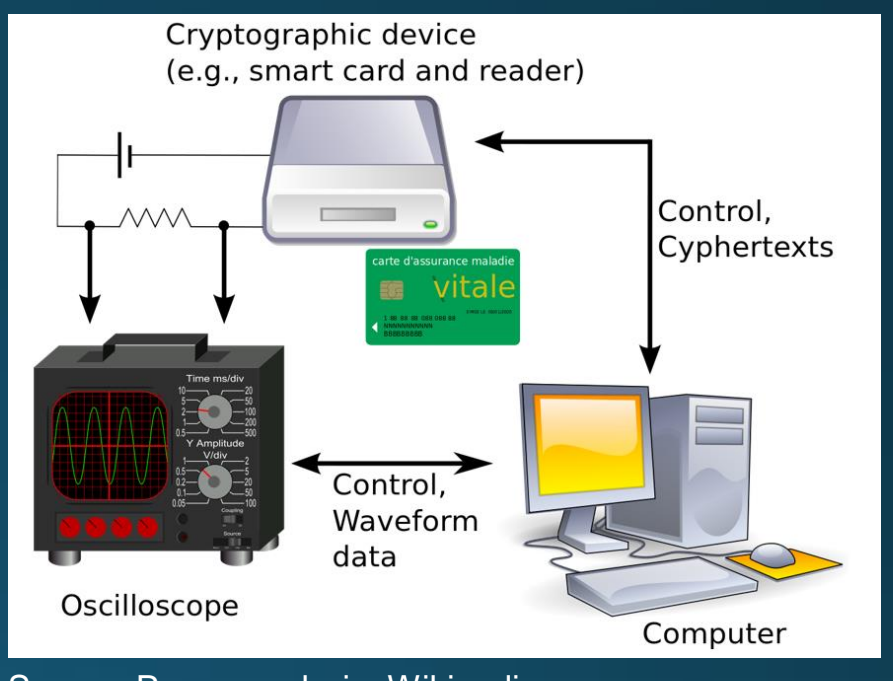

Source: Power analysis, Wikipedia,

https://en.wikipedia.org/wiki/Power_analysis 


\section{Sense Amplifier Based Logic (SABL)}

One of the main Dual-Rail Pre-Charge (DRP) circuitries

口 Complementary signals:

- Input: in and in

- Output: out and $\overline{\text { out }}$

Operating Phases:

- Pre-charge: All signal goes to LOW (0)

$>$ Internal nodes and capacitances all are pre-discharged

- Evaluation: One rail stays at " 0 " and its dual goes to "1"

$\square$ Need a full custom design

- Differential Pull Down Network

Twice area \& power and half clock frequency

$\checkmark$ Avoiding glitches

$\checkmark$ Power $\rightarrow$ independent of the data being processed

$\checkmark$ Significantly hinders PA attacks

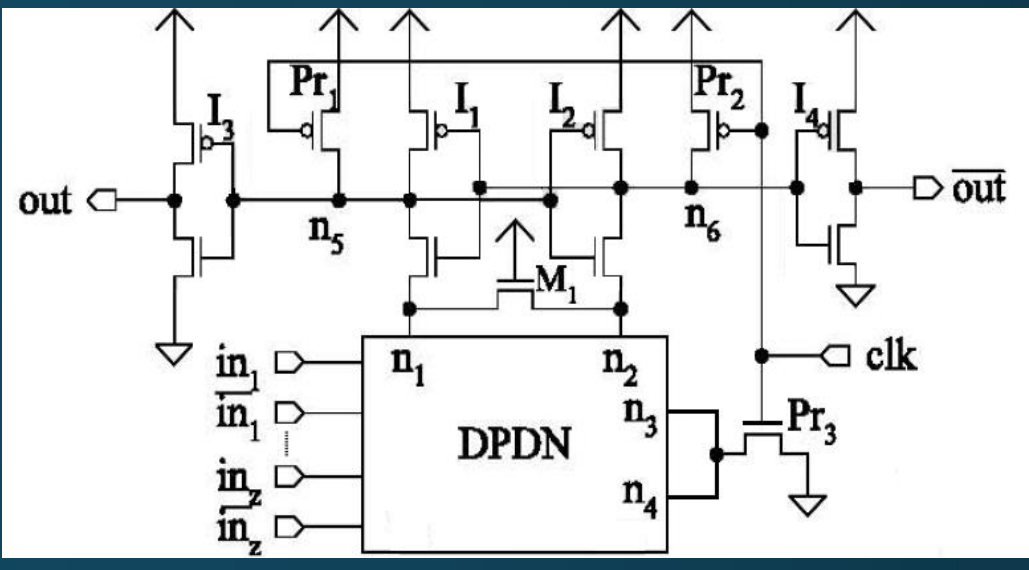

Overall-view of an SABL gate

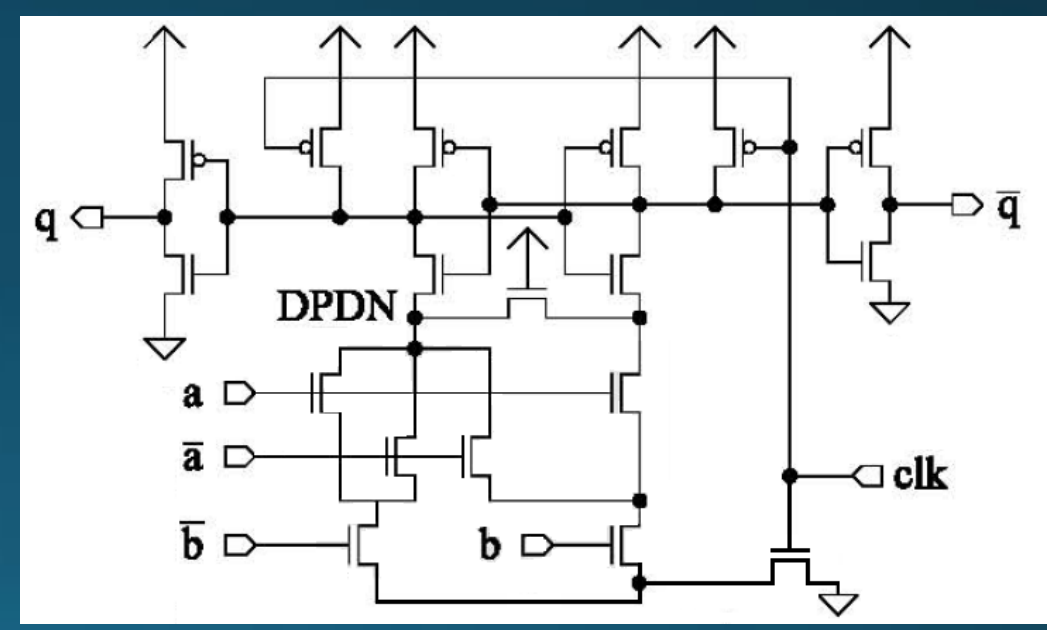

SABLAND/NAND gate

Source: Power Analysis Attacks, Revealing the Secrets of Smart Cards, S. Mangard, E. Oswald and T. Popp 


\section{Device Aging}

Device aging is an important failure mechanism in nanoscale designs that jeopardizes the reliability and/or security of electronic devices

$\square$ Performance of nanoscale CMOS circuits degrades over their lifetime.

\section{Ultimate Failure}

$\square$ Circuit aging mechanisms:

- Bias Temperature-Instability (BTI)

- Hot Carrier Injection ( $\mathrm{HCl})$

- Time dependent dielectric Breakdown (TDDB)

- Electromigration (EM) 


\section{Aging Mechanisms}

$\square$ Negative Bias Temperature Instability (NBTI)

- Cause: Holes creating traps between Si-SiO2 and substrate

- Impact: $V_{\text {th }}$ increase, especially for PMOS transistors

$\square$ Hot Carrier Injection ( $\mathrm{HCl})$

- Cause: Electrons colliding with the gate oxide (rather than going only to the conduction channel between source and drain)

- Impact: $V$ inceas, especially for NMOS transistors

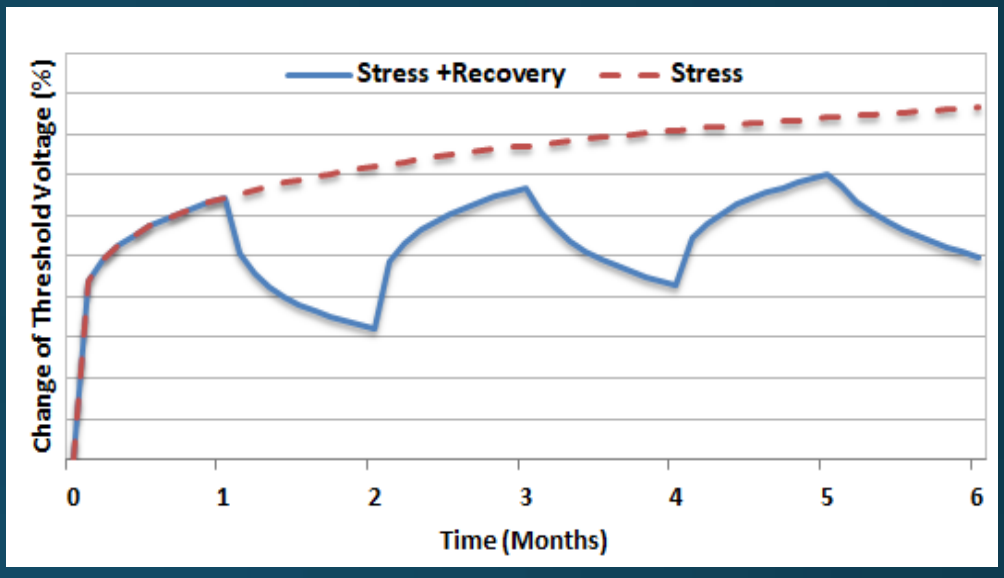

NBTI

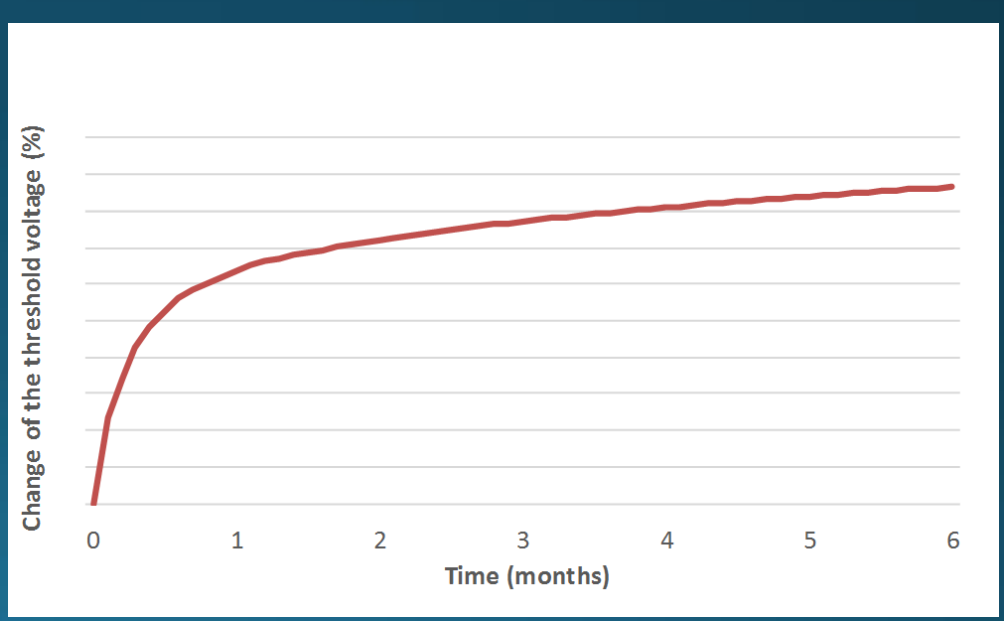

$\mathrm{HCl}$ 


\section{Impact of Aging on $\mathrm{V}_{\text {th }}$ of MOSFETs}

$\square$ The threshold voltage of a MOSFET can be used as a parameter to regulate the trade-off between its propagation delay and its leakage current.

$\square$ Devices with a high threshold voltage are slower and can be used where timing is not critical in order to reduce the leakage current.

$\square$ By aging a CMOS circuit, the threshold voltage of its transistors increases, and the design fails to meet the timing requirements.

The aging process can be accelerated by increasing the supply voltage and operating temperature. 


\section{Aging-induced Delay Change}

$\square$ Fed with the input values that results in maximum NBTI aging

U Unprotected and SABL circuits experience different amount of delay change

- Related to their transistor level topology

Each path in a cryptographic device may degrade with a different rate based on:

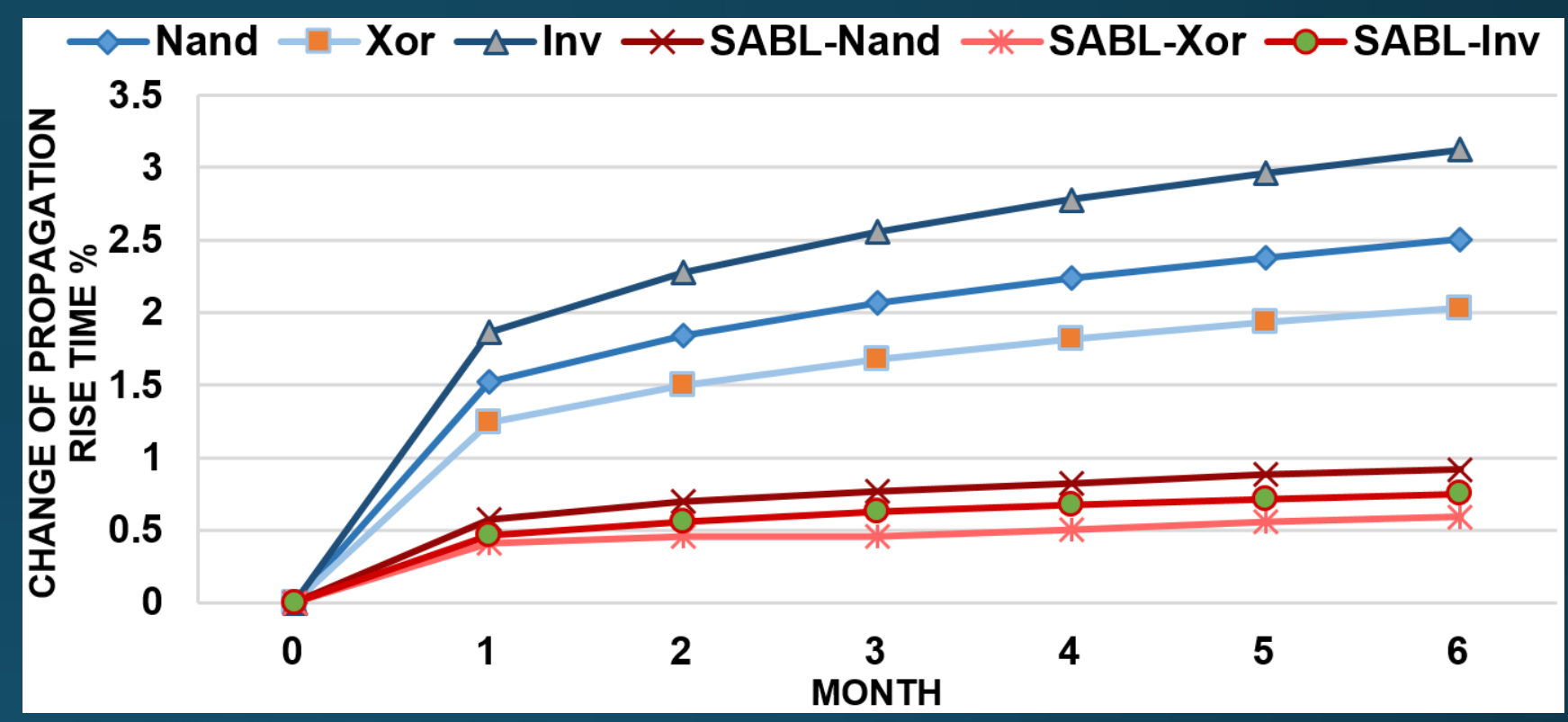

- Type of its underlying gates

- The input values feeding it

* Aging can result in imbalances in the power consumption of SABL circuits.

* These imbalances can increase the success of the PA attacks on such protected devices.

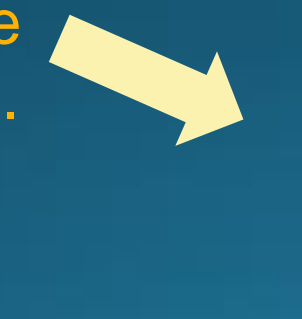

Need to investigate the effect of device aging on the resiliency of SABL-Protected Circuits against Power Analysis Attacks. 


\section{Present Architecture}

Targeted Circuit

$\square$ Nibble-serial PRESENT implementation with single Sbox instance:

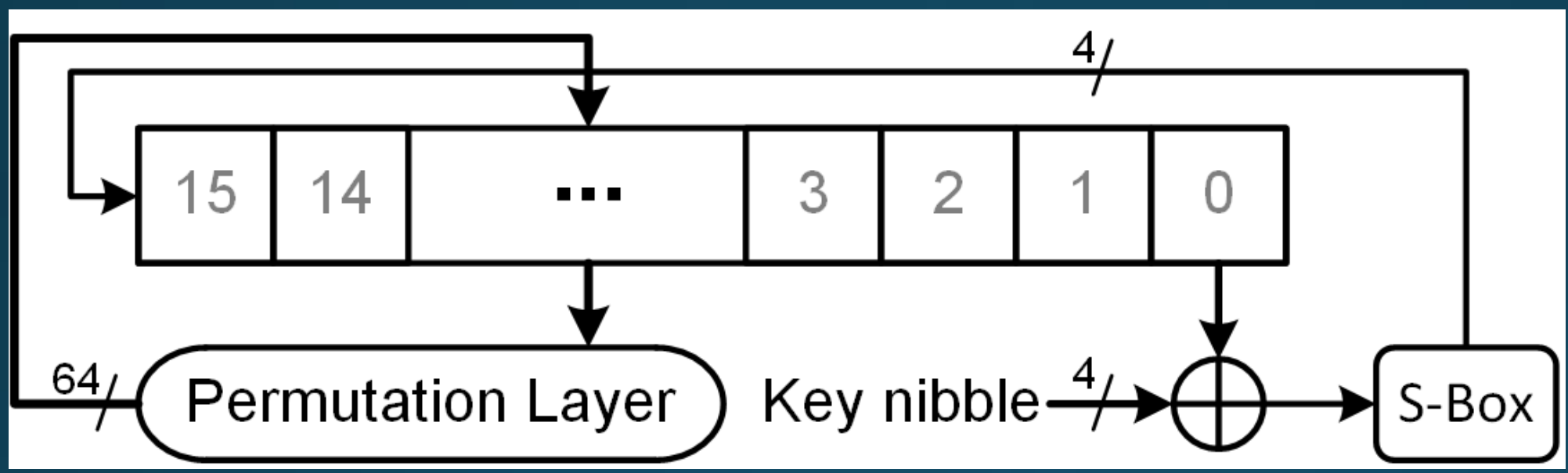

$\square$ Design:

- Area-optimized

- Data paths are serialized to 4-bit words (one nibble)

- Requires 563 clock
Simulation:

- RTL converted to SABL- Dual Rail

- Transistor level simulation

- Includes:

- NMOS: 21,980

- PMOS: 13,838 


\section{Experimental Setup}

Q Technology:

- 45-nm NANGATE library

$\square$ Simulation Voltage and Temperature

- $V_{d d}: 1.2 \mathrm{~V}$

- Temperature: $80^{\circ} \mathrm{C}$

$\square$ Aging Evaluation:

- Deploying Synopsys HSpice MOSRA Level 3 model

- Assessing NBTI and $\mathrm{HCl}$ aging effects

- Aging effects were assessed for 8 weeks of operations (1-week steps)

Attack Setup:

- Present Cipher (S-Box module) operated at $300 \mathrm{MHz}$

- Randomly generated plaintexts and a fixed key

- Targeting first Round: 22 Clock Cycles

- 1665 sample points in each clock cycle 


\section{Power Change: Unprotected circuit \\ Experimental Results}

$\square$ Five current traces corresponding to

- Five different plaintexts

- But the same key

Traces extracted when one S-Box operation is performed.

$\square$ Current traces are deviating from each other in different points of time.

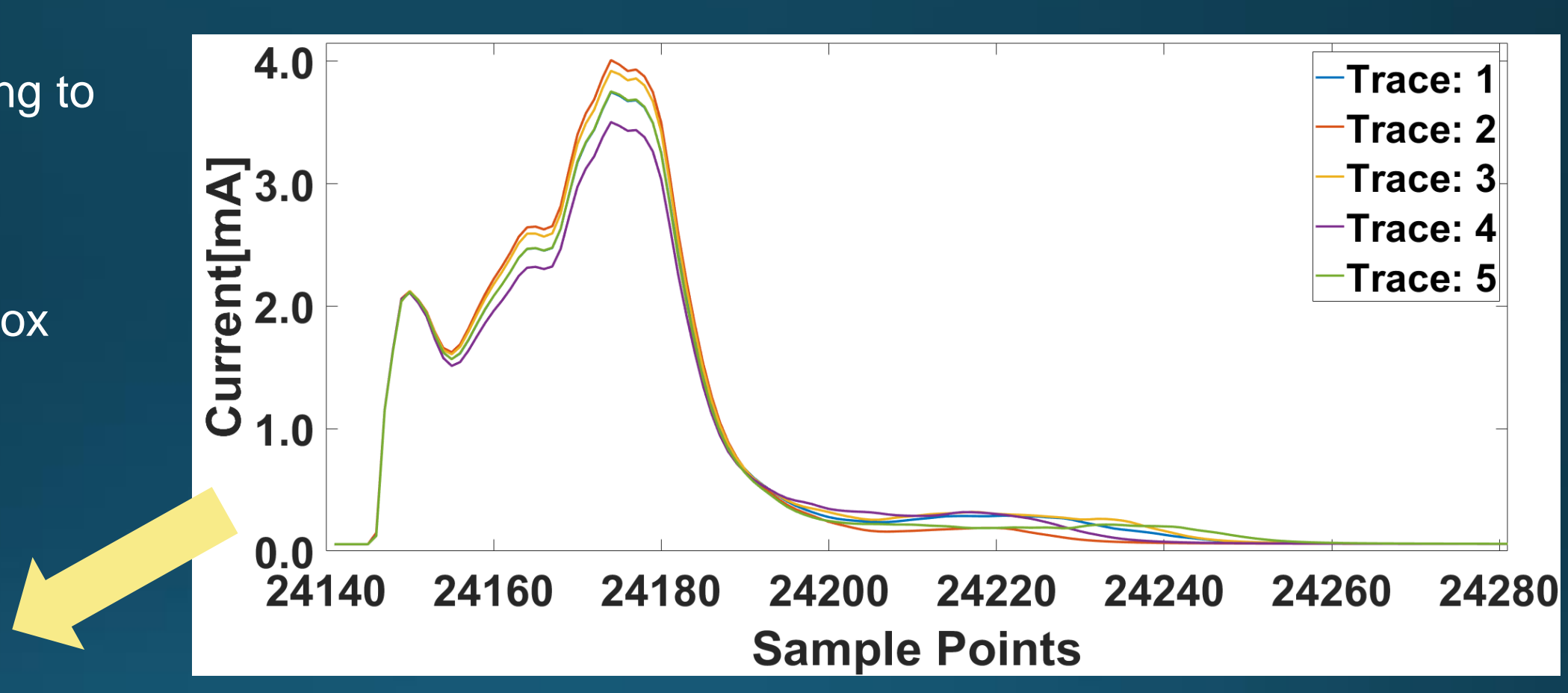

Unprotected circuit (Age $=0$ )

$\square$ The deviations can be

exploited by the adversary to

recover the key. 


\section{Aging-induced Power Change}

\section{Experimental Results}

$\square$ SABL Protected $($ Age $=0)$ :

- The current traces are highly balanced

- This makes the device compromising highly difficult (if not impossible).

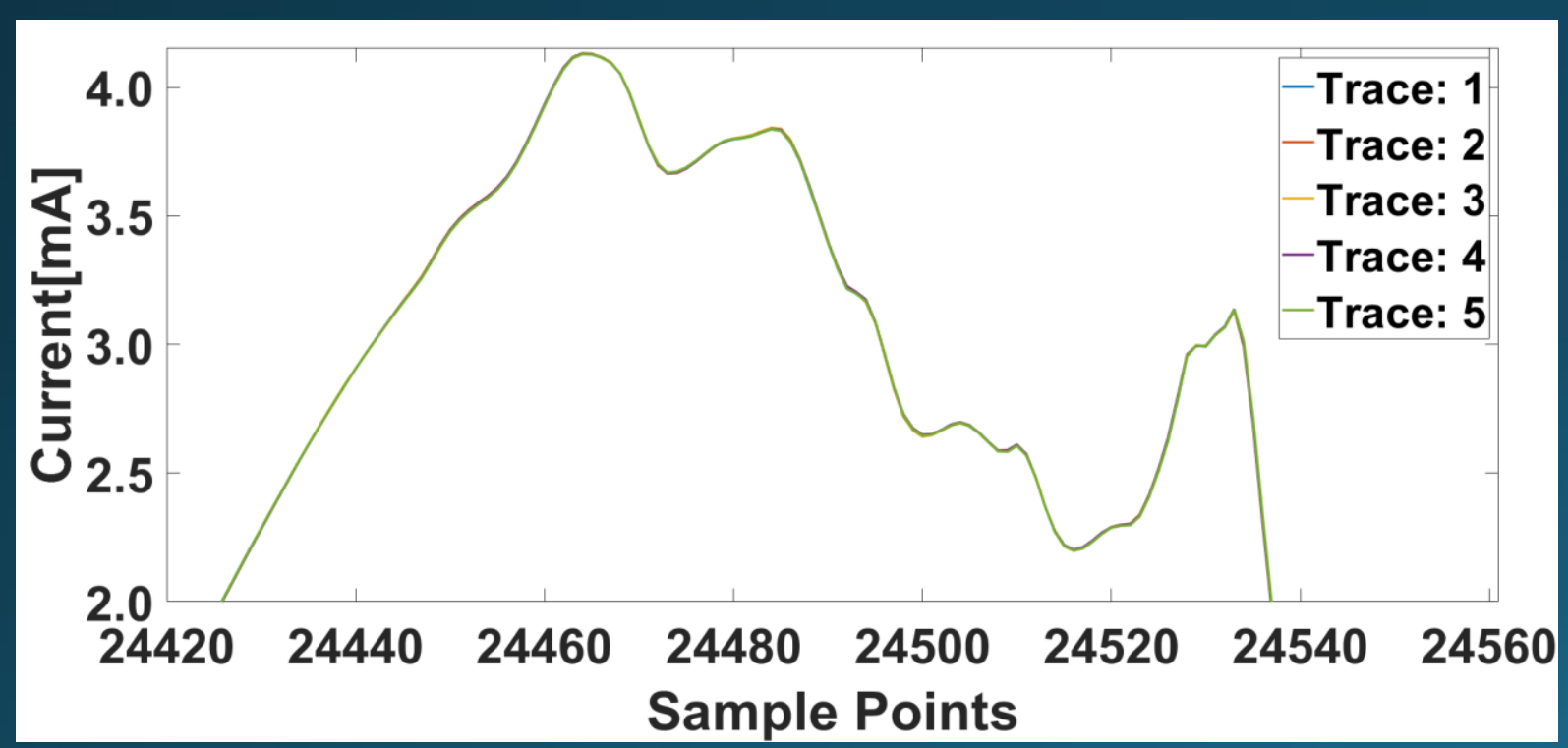

SABL Protected circuit $(\mathrm{Age}=0)$ $\square \mathrm{SABL}$ Protected (Age $=8$ Weeks):

- Deviates from equalized power consumption

- Deviations can be exploited by the adversary to extract the key.

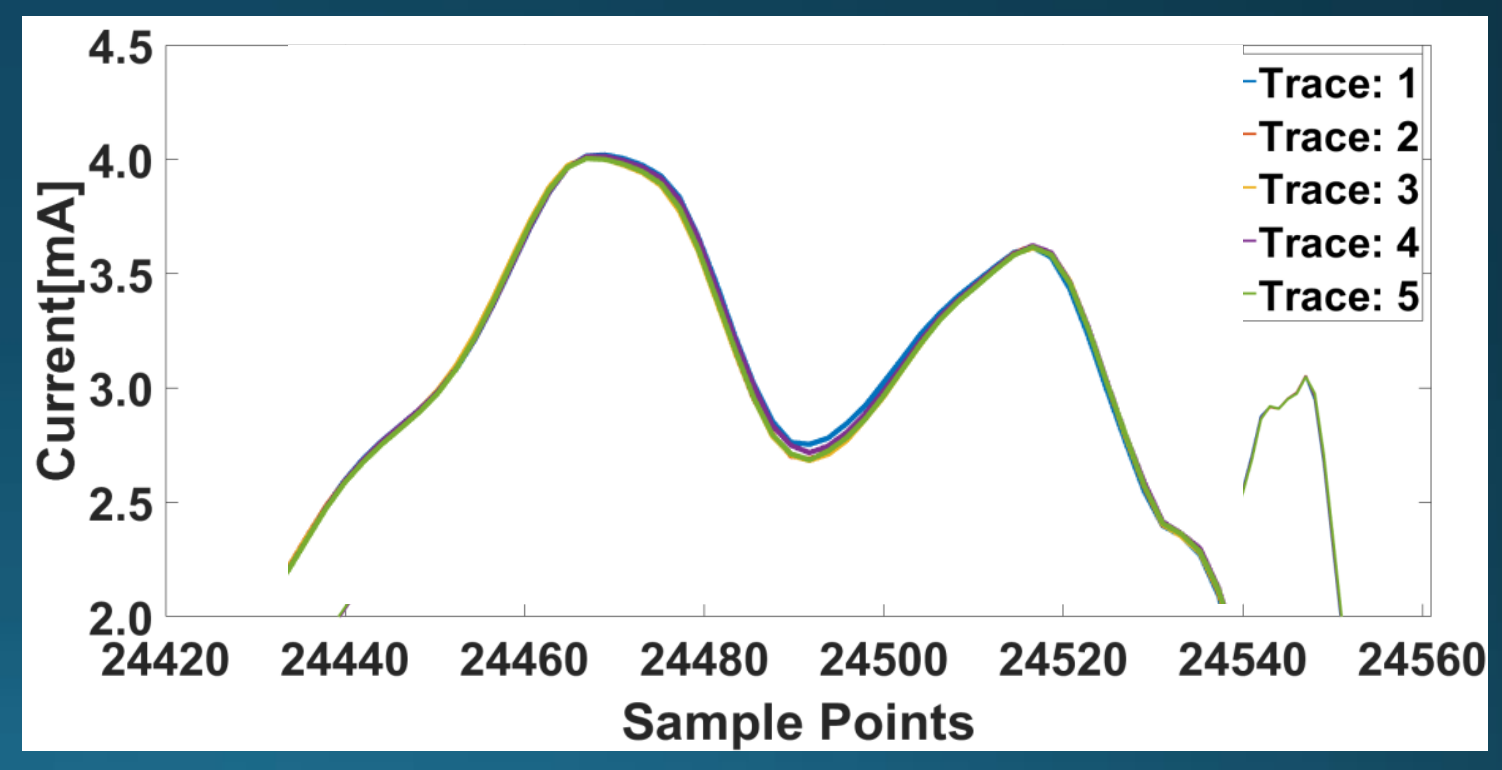

SABL Protected circuit (Age $=8$ Weeks) 


\section{Aging-induced Variance change}

Experimental Results

$\square$ Corresponding variance of several traces for

- New circuit: Week 0

- aged circuit: Week 8

$\square$ With aging, the variance is increased

Increase of leakage with aging

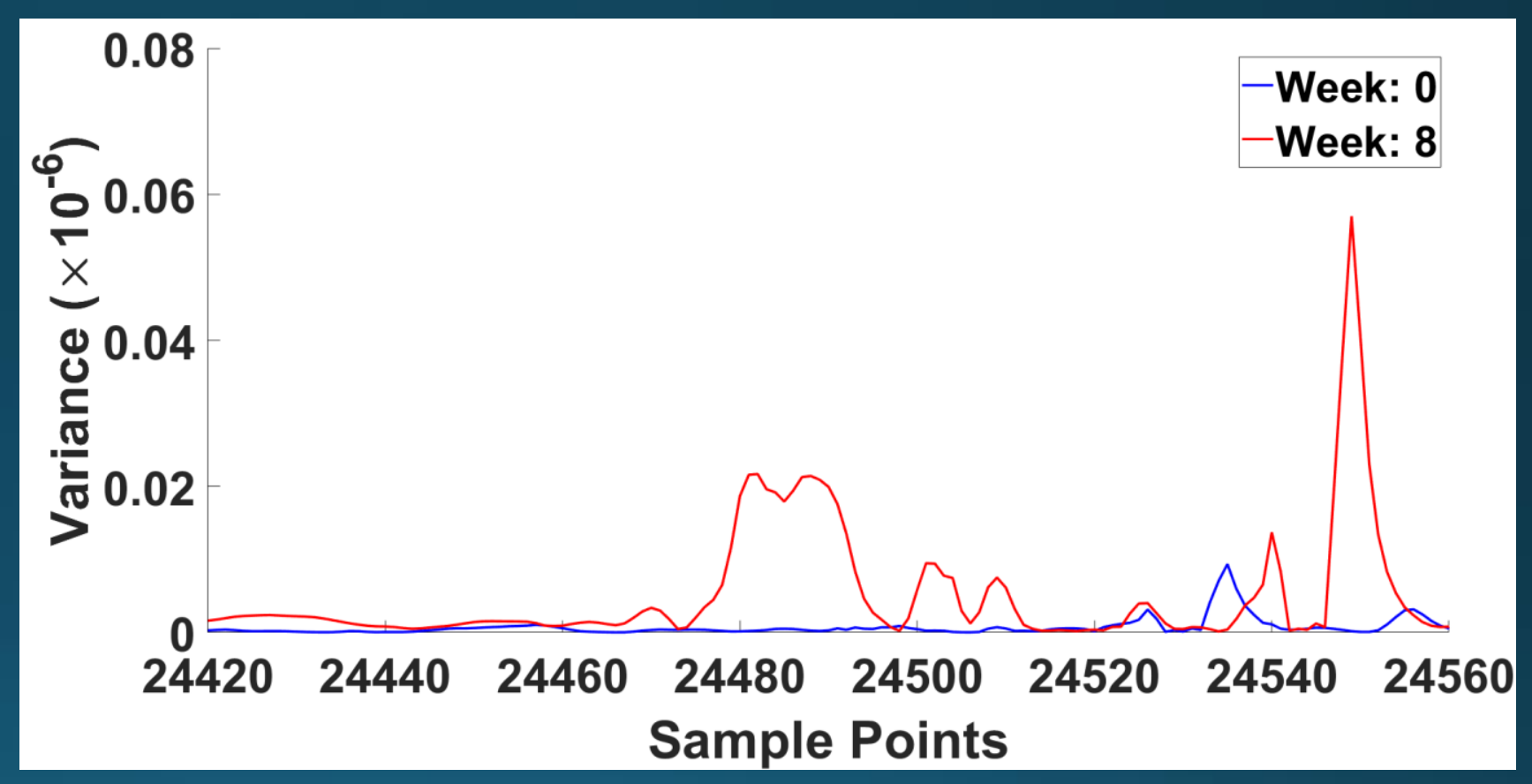

Figure: Variance traces of the SABL-protected circuit. 


\section{Aging-induced Dynamic Power Change}

Experimental Results

$\square$ Dynamic power change during the computation of an S-Box

$\square$ One randomly generated plaintext

Power traces for week 0 to 8

$\square$ Observations:

- Magnitudes of the current trace changes during the device lifetime.

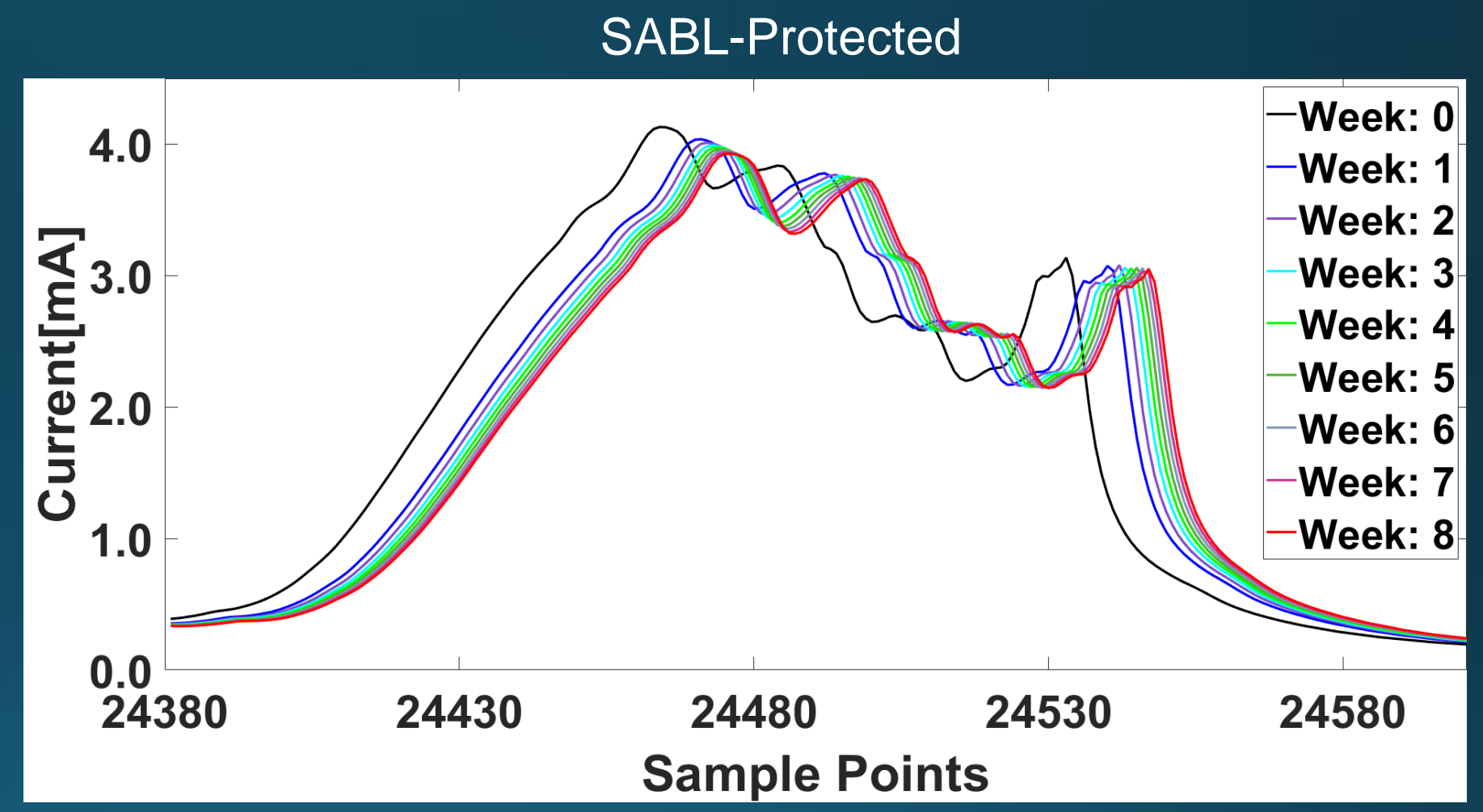

Current traces during the computation of an S-Box for the same input in different aging conditions 


\section{Mutual Information}

Experimental Results

Ml reveals how much noise is required to avoid the exploitability of leakages.

$\square$ Observations:

- Ml drops with a higher amount of noise when the circuit is aged

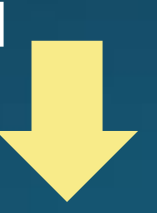

- Information leakage is increased by aging in SABL circuits

\section{SABL-Protected}

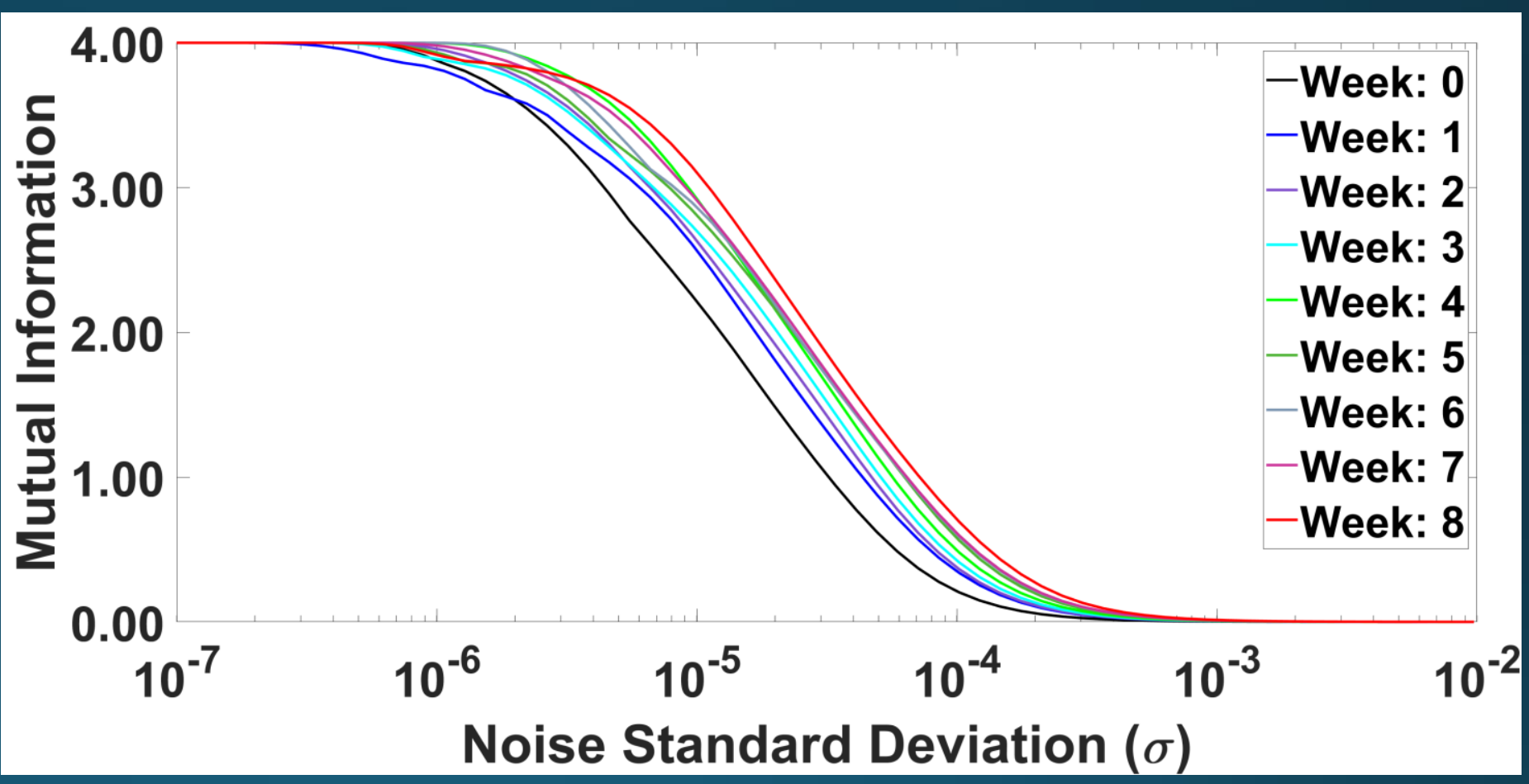

Figure: Mutual Information curves associated to an S-Box input for different aging conditions. 


\section{Power Analysis Attacks}

\section{Experimental Results}

Side-channel leakage was analyzed with Correlation Power Analysis (CPA)

- Hypothetical model to an intermediate value that depends on

* Known input as well as (part of) the unknown key

$\square$ Attack Setup:

- Traces without Noise: 90

- Added noise: 100 times

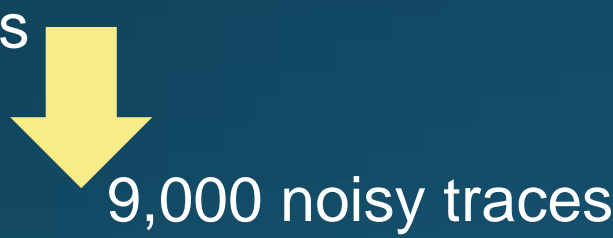

- Repeated each attack and evaluation 50 times to remove randomness

- Power Model: Hamming weight (HW)

Suits best as the signals are set to 0 in pre-charge phase of SABL, then evaluation phase starts 


\section{PA Attacks: CPA Results}

\section{Experimental Results}

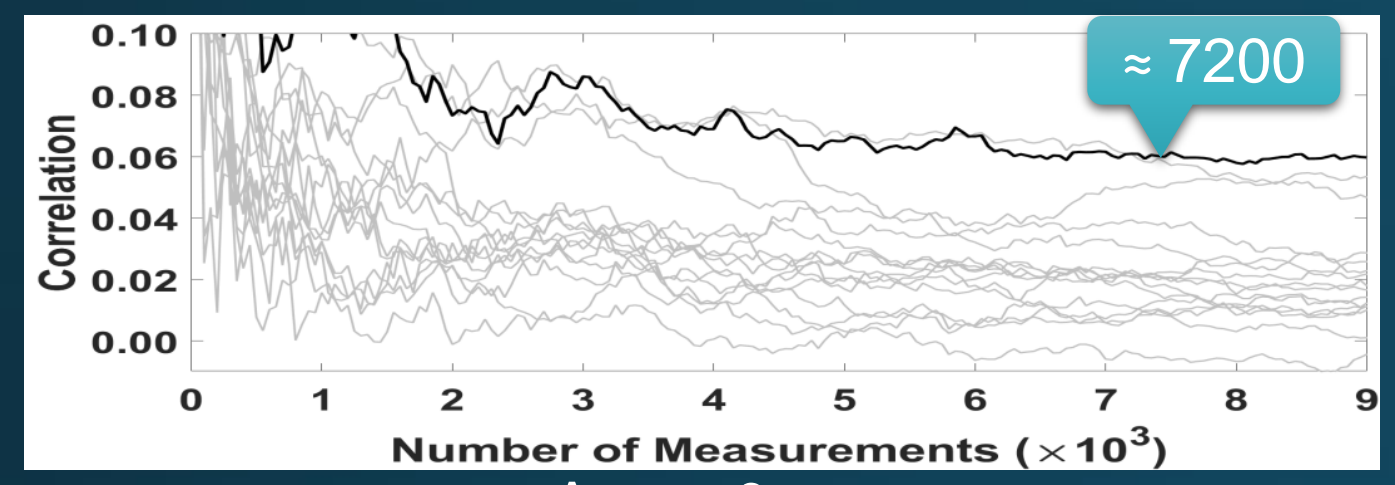

Age $=0$

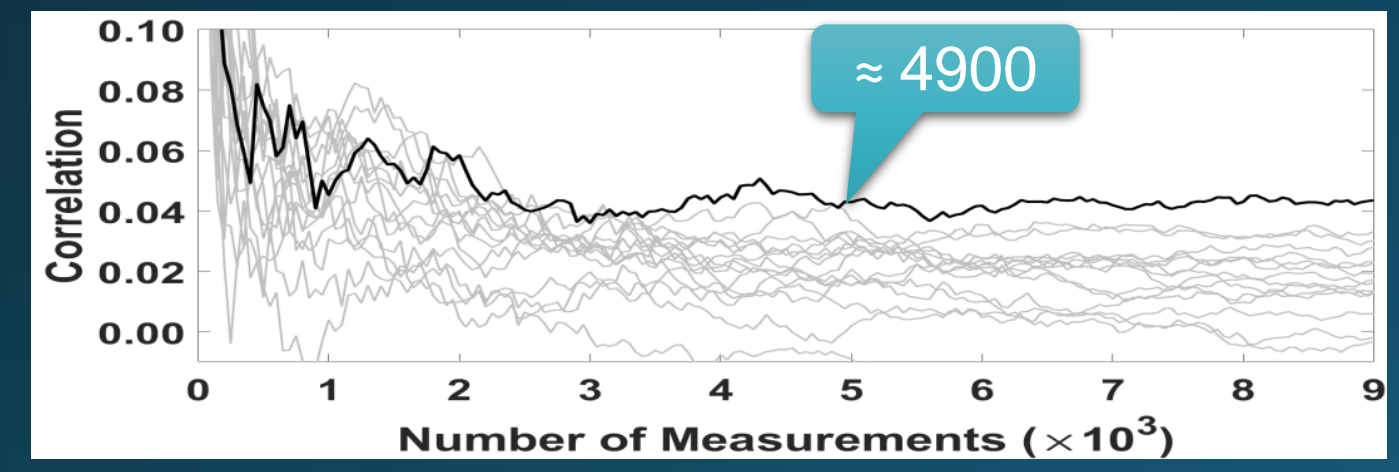
Age $=5$ Weeks

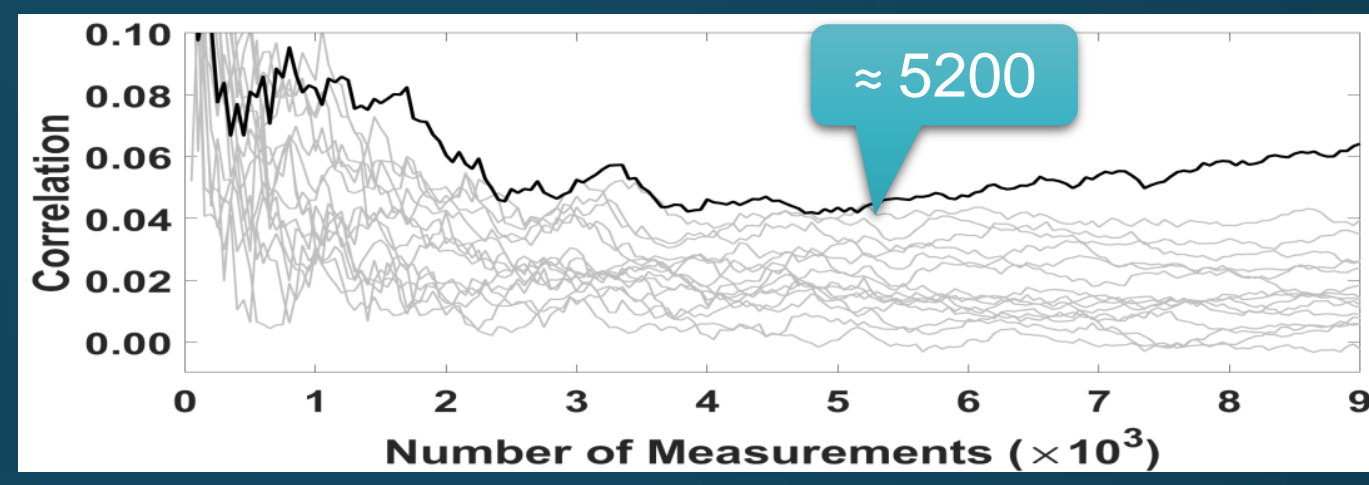
Age $=2$ Weeks

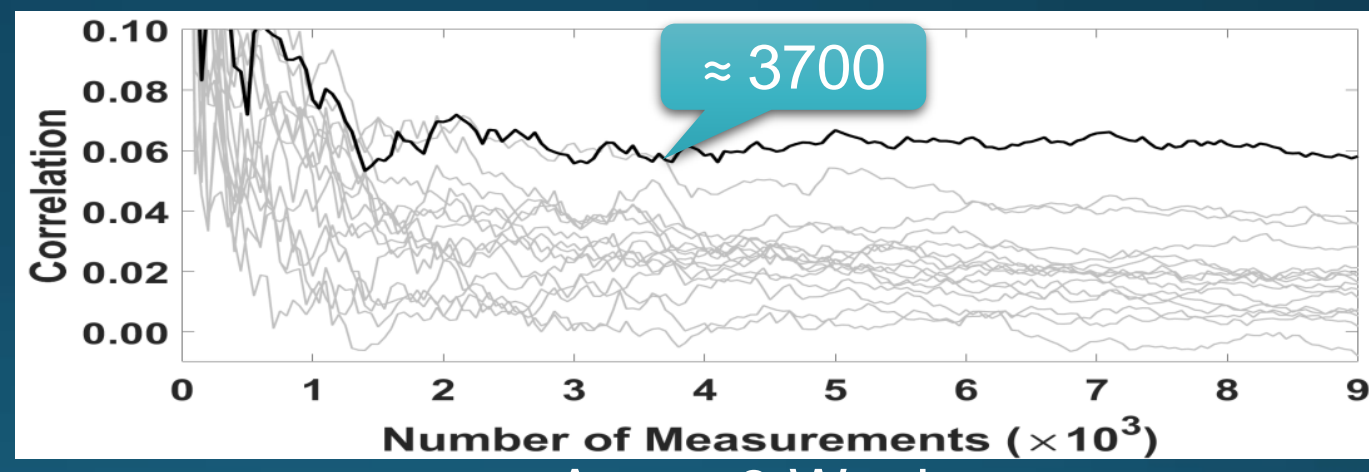
Age $=8$ Weeks

* Due to Aging: SABL-protected circuit experiences imbalances

Aged circuits Need less traces for a successful attacks 


\section{PA Attacks: Success Rate}

\section{Experimental Results}

口 CPA attack with HW model

$\square$ Average success rate of the attack targeting all key nibbles

$\square$ Success Rate:

- Week 0: $\approx 81.4 \%$

- Week $1: \approx 86.4 \%$

$\square$ Confirming that device aging makes the attack easier on SABL circuits.

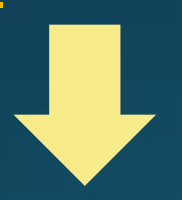

$\square$ Necessity of revisiting the security of power-equalized cryptographic devices with

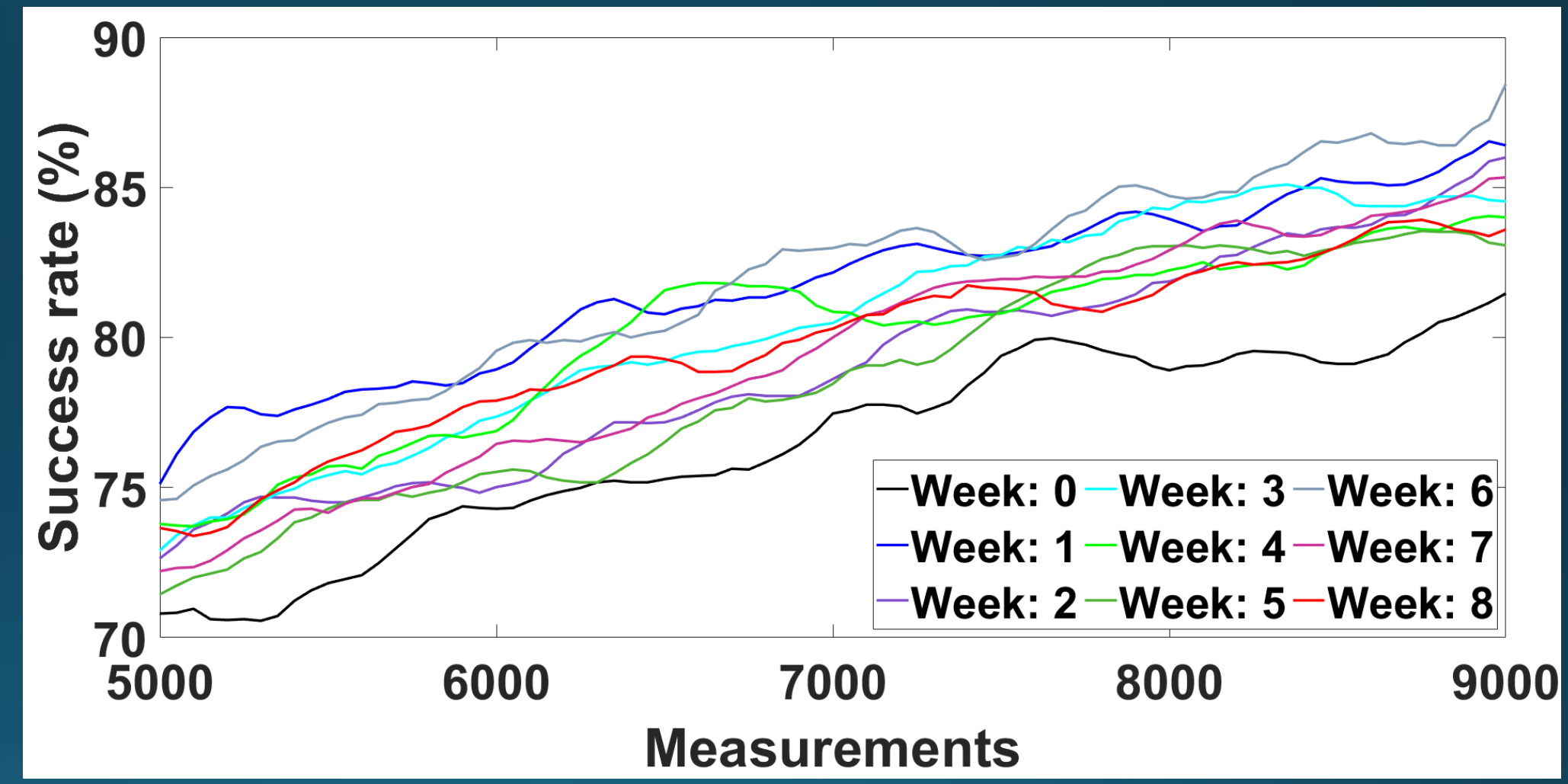
respect to aging 


\section{Conclusion and Future Directions}

$\square$ Investigated the impact of transistor aging on the success of power analysis attacks launched on the SABL-protected PRESENT cipher.

$\square$ Device aging defeats the purpose of the SABL countermeasure, and results in power consumption imbalances originated from the change of transistors' threshold voltage over time.

For the first time, we illustrated that such deviation of transistors specification over time can ease the attacks launched on SABL protected circuits.

$\square$ Success rate increased over $6.1 \%$ (from $81.4 \%$ to $86.4 \%$ ) when targeting a 1 -week-old device.

$\square$ This observation calls for proper secure implementations of cryptographic circuits that are resilient against power analysis attack over the circuit lifetime regardless of its age.

* Future Directions:

Investigating the impact of device aging on the success of the attacks that exploit static power in SABL circuits

$\square$ Validating our findings on real silicon.

$\square$ Designing an aging-resilient SABL-Based circuitry to provide lifelong security for cryptographic devices 


$$
\begin{aligned}
& \text { Thanks for your attention. } \\
& \text { Any questions? }
\end{aligned}
$$

